$$
\text { CENF } 9210.246 \ldots 1.3
$$

LA-UR-93-0043
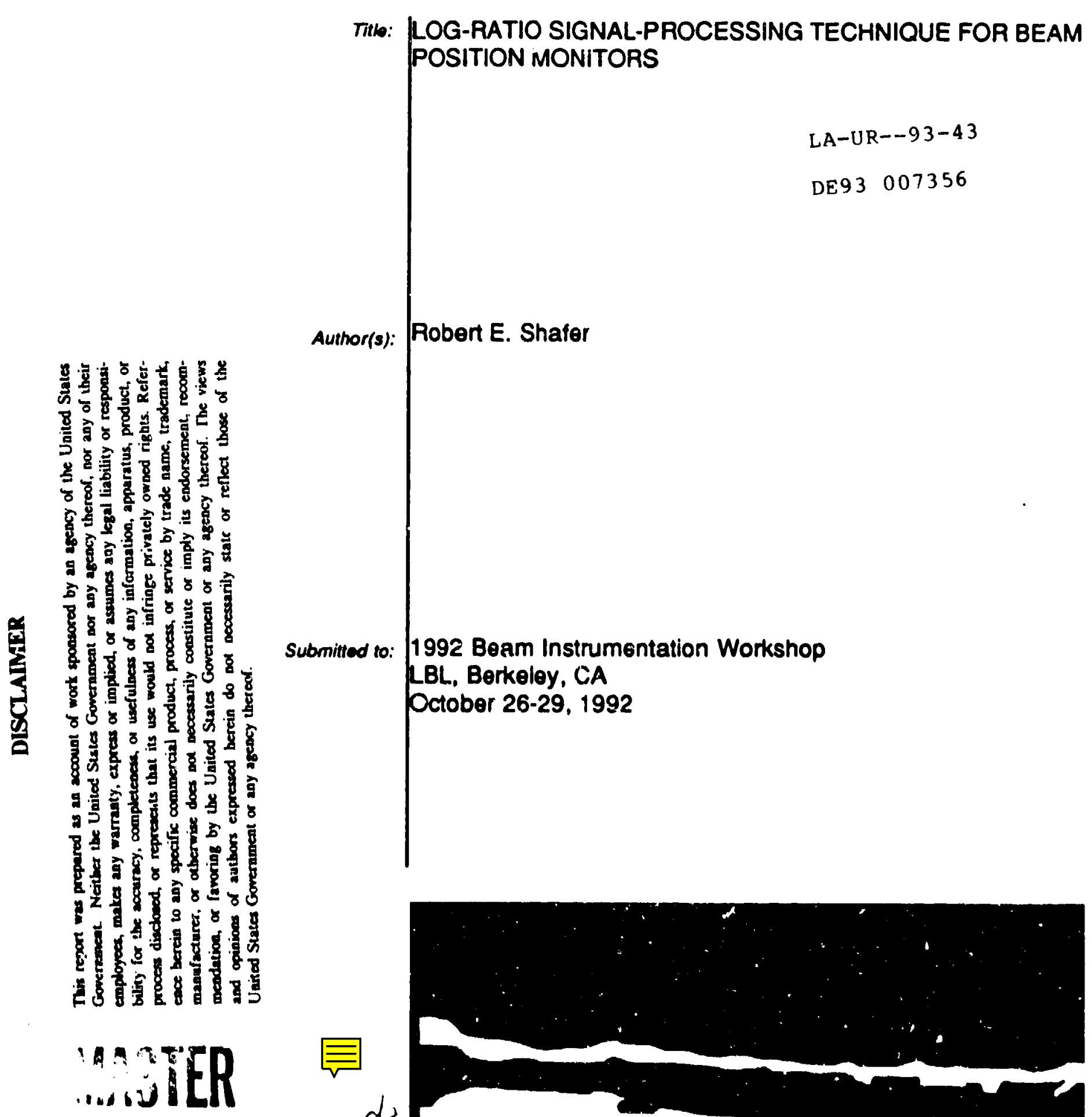

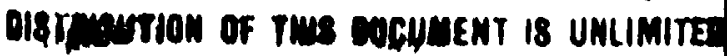

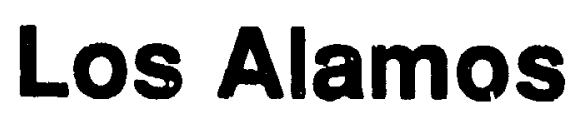

NATIONAL LABORATORY

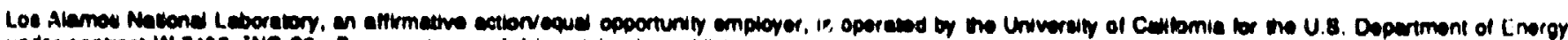

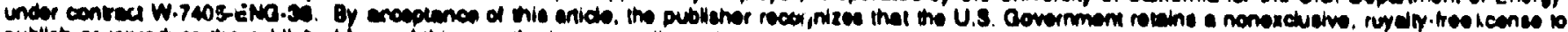

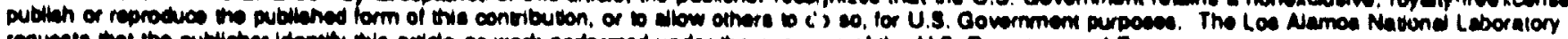

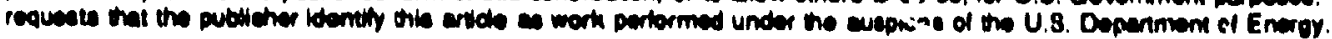




\title{
LOG-RATIO SIGNAL-PROCESSING TECHNIQUE FOR BEAM POSITION MONTTORS
}

\author{
R. E. Shafer \\ Los Alamos National Laboratory \\ Los Alamos, NM 87545
}

\section{INTRODUCTION}

Two basic signal-processing techniques are presently in wide use for the processing of signals from beam position monitors (BPMs); difference-over-sum, and amplitude-modulation-to-phase-modulation (AM-PM) conversion. Difference-oversum offers simplicity and low cost, but poor real-time normalized response and amplitude dynamic range. AM-PM offers fast real-time response and large dynamic range, but is costly and difficult to implement. Logarithmic-ratio processing, a technique using newly available inexpensive hybrid circuits, appears to offer the advantages of both, and the disadvantages of neither. This paper reviews the features of all three processing techniques, and highlights the features of the log-ratio technique. Among the advantages of log-ratio is a beam-displacement response linearity that is superior to either difference-over-sum or AM-PM for circular-aperture BPMs.

\section{SIGNALS FROM BEAM POSITION MONITOR ELECTRODES}

Signals from most beam position monitors, including both buttons and directionalcoupler or stripline pickups, are not linear in the transverse beam displacement from the BPM axis. For a pencil-beam current $I_{\mathrm{b}}$ at position $r, 6$ in a circular-aperture BPM of radius $b$ and electrode-width $\phi$ as shown in Fig. 1, the electrode response is given by 1

$$
I_{R}=\frac{-I_{b} \phi}{2 \pi}\left\{1+\frac{4}{\phi} \sum_{n=1}^{\infty} \frac{1}{n}\left(\frac{f}{b}\right)^{n} \cos (n \theta) \sin \left(\frac{n \phi}{2}\right)\right\}
$$

and

$$
L_{L}=\frac{-I_{b} \phi}{2 \pi}\left\{1+\frac{4}{\phi} \sum_{n=1}^{\infty} \frac{1}{n}\left(\frac{L}{b}\right)^{n} \cos (n \theta) \sin \left(n\left[\pi+\frac{\phi}{2}\right]\right)\right\}
$$

The beam position displacement response of a pair of electrodes is best characterized by the $\mathrm{dB}$-ratio of the two electrode signals:

$$
\left(\frac{R}{L}\right)_{\mathrm{dB}}=20 \log _{10}\left(\frac{R}{L}\right)=\frac{160}{\operatorname{Ln}(10)} \frac{\sin (\phi / 2)}{\phi} \frac{x}{b}+\text { higher onder terms }
$$

where $R$ and $L$ represent the right and left electrode signal amplitude outputs, and $x=r \sin \theta$. Certain special geometry electrodes can provide a linear response to 
beam displacement, but at the expense of sacrificing the high-frequency response characteristics of the electrodes. A diagonally-cut electrode pair is a good example.

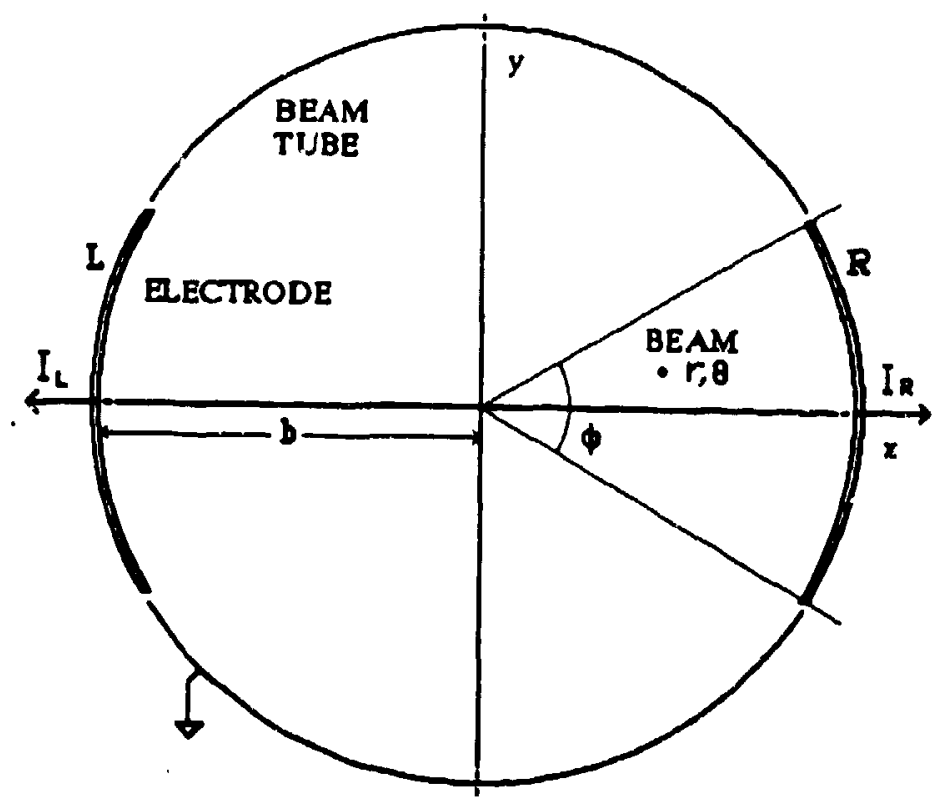

Fig.1. Cross section of a circular-aperture beam position monitor.

\section{BPM SIGNAL PROCESSING CONSIDERATIONS}

In designing a signal processing system for use with BPM signals, a wide variety of features must be considered. Some of the more important considerations are listed below.

1. Frequency-domain vs, time-domain processing. A wide-band processing system generally processes BPM signals in the time domain, while a narow-band system can process a single Fourier hanuonic compinnent of the rf-modulated BPM signal, thus allowing the processing to be carried out in the frequency domain. There is more available signal power in the wide-band processing system, but because of signal attenuation and dispersion in cables, some of the temporal information (e.g., pulse shape) is distorted. In narrow-band processing, signal dispersion is not a concern, but there is less available signal power (and less kTB noise). Some signal-processing methods are best suited for frequency-domain processing, while others are equally suited for both time- and frequency-domain processing. Frequency-domain processing can be used with individual isolated beam bunches as well as $\mathrm{cw}$. nodulated beams.

2. Processing frequency Generally, processing of frequency-domain signals at the beam-bunching frequency is the most straight forward. Signal power from the electrodes is very frequency dependent, depending on both the particle bunch length and electrode length. For accelerators with high-bunching frequencies (e.g., $425 \mathrm{MHz}$ ), signal down-conversion to an intermediate frequency must be 
considered, due to limitations of the electronics. AM-PM processing at an intermediate frequency is also required when the beam-bunching freciuency varies with time, such as in a low-energy proton synchrotron. In this case, the local oscillator must track the if frequency.

3. Signal dynamic range: The signal power output from the BPM electrodes varies quadratically with the beam current. Any signal-processing system that processes the electrode signals directly must be able to handie this required dynamic range in signal power. Furthermore, any $\mathbf{R}-\mathrm{L}$ (difference) signal of the two opposite $\mathbf{R}$ and $L$ (for right and left) electrodes generally also varies nonlinearly with the beam displacement, so the total electronics dynamic range must be quite large. For example, a system designed to handle a 100:1 range of beam current and achieve resolution of about $10^{-3}$ of the aperture needs a linear dynamic range of nearly $10^{5}$ to 1 . This dynamic range is not achievable for un-normalized signals without range switching.

4. Normalized response If the processed analog signals are of the form $R / L$. (i.e., ratio) or $(R-L) /(R+L)$ (difference-over-sum), then the signal amplitude is independent of beam current, which reduces the electronics dynamic-range requirement. Both AM-PM and log-ratio processing provide normalized output response.

5. Real-time response. Some applications of BPM cignal processing systems require fast real-time normalized response. If the electrode signals are separately digitized, and the normalization is performed digitally, there is a long delay time between the signal acquisition and the available normalized beam position signal. Both AM-PM and log-ratio processing can provide the normalized real-time signal without off-line processing.

6. Displacement response linearity No BPM signal processing technique can provide normalized signals from stripline or button electrodes that are completely linear in beam displacement. The natural nonlinearity in some signal processing techniques can compensate for this, however.

7. Cable phase-matching Some signal ocessing techniques require close phase matching of the signals from the individuai electrodes. Generally the AM-PM processing method requires phase matching to about \pm 5 degrees of phase at the processing frequency. This is also thue of difference-over-sum processing if the difference and sum $(R-L$ and $R+L)$ signals are formed prior to the if envelope detection.

8. Accuracy and resolution. Absolute position accuracy is usually limited to about $1 \%$ of the BPM half-aperture, unless special techniques are used to monitor and correct the channel-to-channel gain disparity. This includes self-calibration procedures that measure both the cable attenuation and electronics drifts. BPM position resolution on the other hand can achieve $\pm 0.01 \%$ of the half-aperture or better, depending primarily on the signal-to-noise ratio, which is limited by thermal noise and by the electronics noise figure. 


\section{COMPARISON OF SIGNAL PROCESSING METHODS}

For two opposing stripline or button electrodes providing output electrical amplitudes $R$ and $I$. (representing $I_{R}$ and $I_{L}$ ), the three signal processing techniques provide the following normalized (meaning independent of beam current) output responses: ${ }^{1}$

Processing Technique

Difference-over-sum

AM-PM

Log ratio

\section{Signal Processing Amplitude}

$$
(\mathbf{R}-\mathbf{L}) /(\mathbf{R}+\mathbf{L})
$$

$$
\tan ^{-1}(R / L)-\pi / 4=\tan ^{-1}[(R-L) /(R+L)]
$$

$$
0.5^{*} \operatorname{Ln}(R / L)=\tanh ^{-1}[(R-L) /(R+L)]
$$

Thus the linearity of AM-PM ard log-ratio processing is essentially that of a nonlinear difference-over-sum response. For a circular-aperture BPM with 45-degree electrode widths, the signal processing responses to a displaced beam are shown in Fig. 2. It is apparent that the log-ratio processing technique has the most linear overall response to beam displacement.

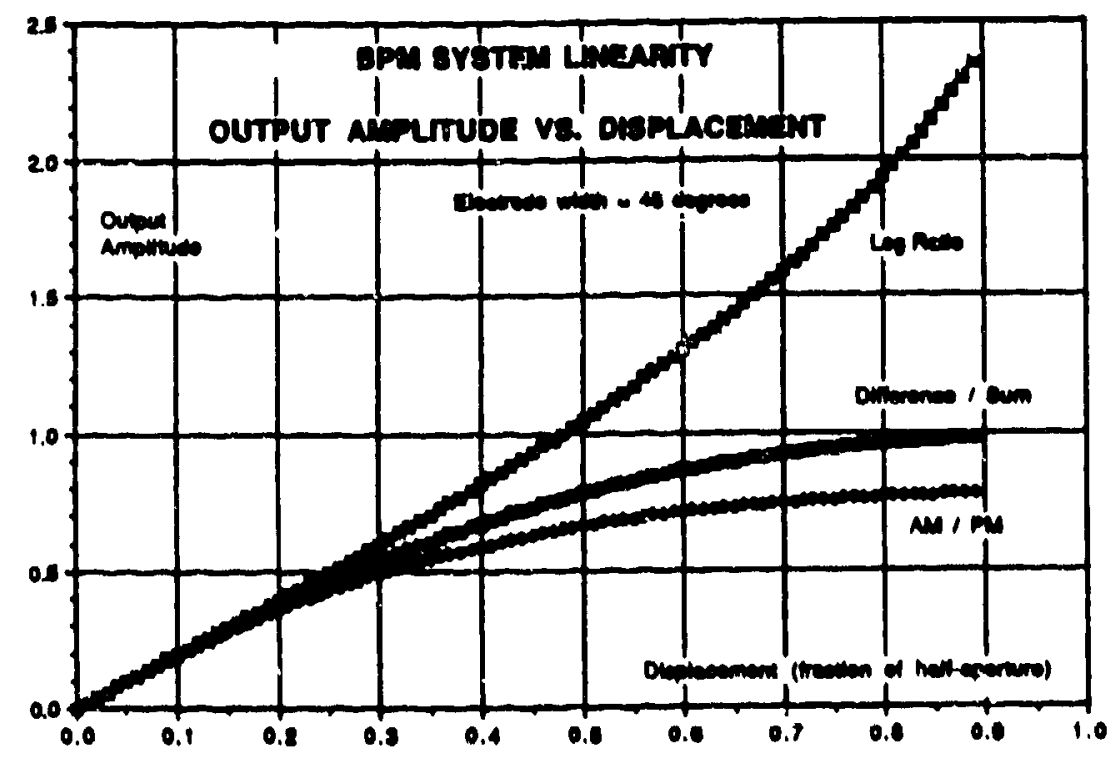

Fig. 2. Signal uutput response to a displaced beam in a circular-apeiture BPM with 45-degree wide electrodes, for difference-over-sum, AM-PM, and log-ratio processing electronics. Log-ratio processing has t'ie most linear response.

Of the three methods, difference-over-sum processing is the simplest and least expensive. The $R$ and $L$ signals can either be combined to create the difference and sum signals prior to or after $\mathrm{ff}$ signal envelope detection. The detecied $R$ and $L$ signals can even be digitized directly, and the sunı and differences calculated digitally. There are two main shortcomings in the difference-over-sum processing method. First, 
processing the $R, L, R-L$, or $R+L$ signals separately requires a large amplitude dynamic range, especially of the $R-L$ signal. Secondly, producing the ratio of $R-L$ to $R+L$ necessary to normalize the signals is difficult in real-time, in either analog or digital processing. Digital processing methods necessarily introduce LSB (leastsignificant-bit) $A D C$ errors that limit the ultimate resolution.

Amplitude-to-phase conversion (AM-PM) processing ${ }^{2}$ uses the two in-phase signals from the BPM electrodes in a circuit that converts the amplitude disparity to a phase disparity. The two resultant output signals have the dependence on the beam current removed by means of a matched pair of hard limiters that preserve the phase information. A double-balanced mixer (or an exclusive OR) is then used to develop a signal proportional to the phase disparity. A typical block diagram is shown in Fig. 3.

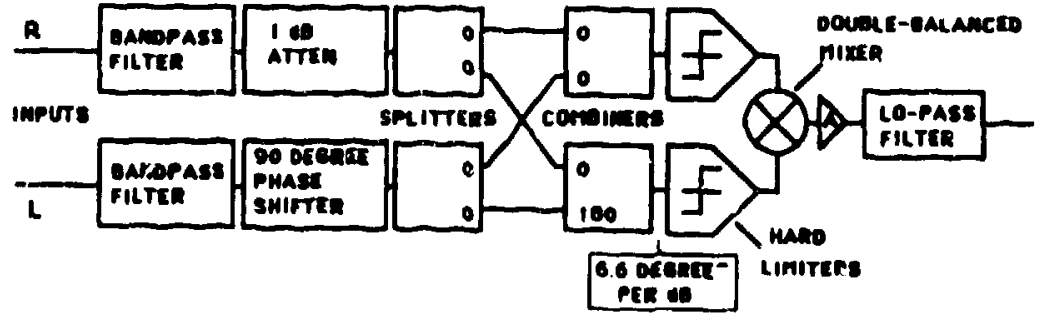

Fig. 3. Block diagram of an amplitude-modulation-to-phase-modulation (AM-PM) conversion signal processing system. Amplitude disparities are converted to phase disparities, which are measured in the double-balanced mixer.

The original log-ratio circuits were based on the exponential V-I relationship of semiconductor junctions. Specifically, for a forward-biased bipolar junction:

$$
I=I_{0}\left\{\exp \left[\frac{e V}{k T}\right]-1\right\}
$$

or

$$
V=\frac{k T}{e} \operatorname{Ln}\left(\frac{I}{I_{0}}\right)
$$

where $I_{0}$ is a characteristic (the reverse-polarity saturation current) of the specific scmiconductor junction, and $\mathrm{kT} / \mathrm{e}$ is about $25 \mathrm{mV}$ at room temperature. An operational amplifier can use a seniconductor junction in a feedback loop as shown in Fig. 4 and achieve a logarithmic response. Because of the dependence of $\mathrm{kT} / \mathrm{e}$ on temperature. and the dependence of $I_{0}$ on the junction characteristics, these amplifiers usually should be used in pairs.

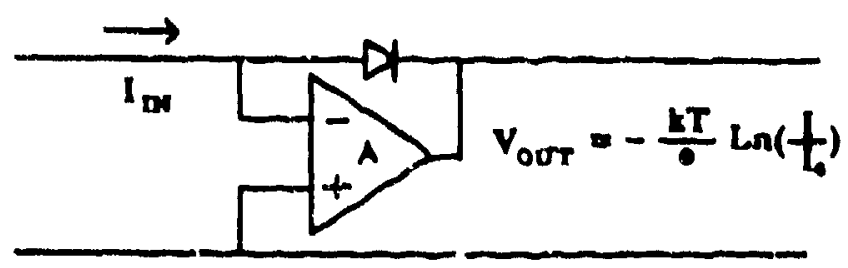

Fig. 4. Simple amplifier circuit with logarithmic response. 
A log-ratio amplifier uses a matched pair of forward-biased diode junctions to match the reverse saturation currents. In this case, the output response is of the form

$$
V_{\text {out }}=G \frac{k T}{e}\left[\operatorname{Ln}\left(\frac{I_{R}}{I_{0}}\right)-\operatorname{Ln}\left(\frac{I_{L}}{I_{0}}\right)\right]=G \frac{k T}{e} \operatorname{Ln}\left(\frac{I_{R}}{I_{L}}\right)
$$

where $G$ is the gain of the output stage (a difference amplifier). Even in this case, the temperature dependence of $\mathrm{kT} / \mathrm{e}$ must be compensated with a thermistor in the output circuit. Because real diodes have a poor ideal diode response, the best semiconductorjunction log-radio amplifiers are constructed using the base-emitter junctions of bipolar transistors as shown in Fig. 5.

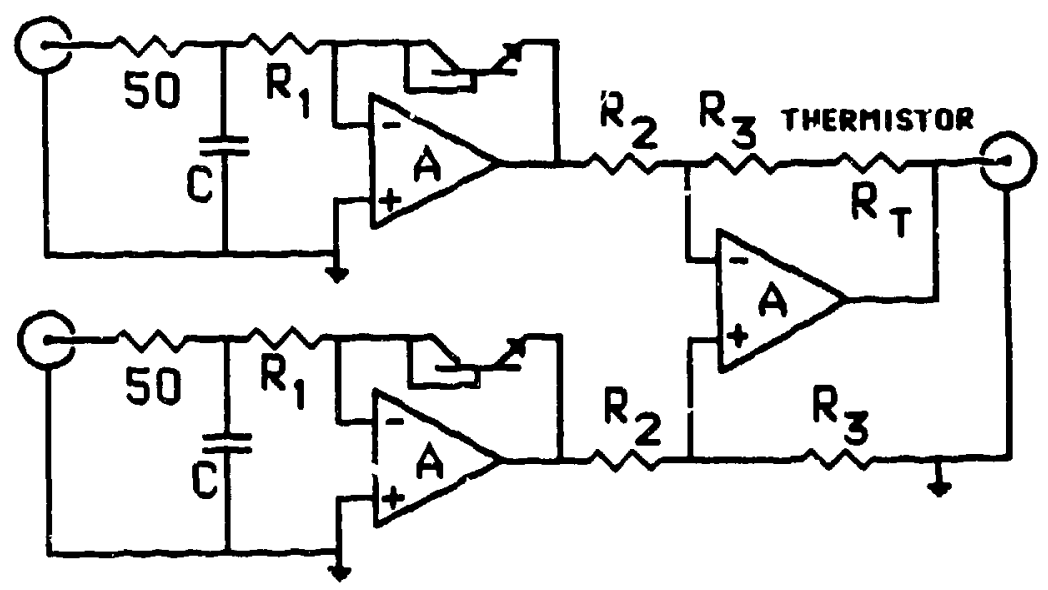

Fig. 5. Log-ratio signal processing system using NPN transistors in a trans-diode configuration. The input circuit is a 50-ohm termination followed by a low-pass filter into the summing junctions. The thermistor is required to sorrect the temperature dependence of the semiconductor junction dynamic resistance. Junction capacitance (about $5 \mathrm{pF}$ ) limits the bandwidth. This circuit can only process unipolar signals.

There are two additional parameters that limit the usefulness of these log amps, however. One is that thie circuits are unipolar, i.e., the input voltage or current cannot be an ac signal. The second limitation is the available bandwidth. (5):

We can define the dynamic impedance of a diode junction as the derivative of Eq.

$$
R_{\text {dynamir }}=\frac{\mathrm{dV}}{\mathrm{dI}}=\frac{\mathrm{KI}}{\mathrm{eI}}=\frac{25 \mathrm{mV}}{\mathrm{I}} \text { ohins }
$$

Thus at $I=1 \mathrm{~mA}$, the dynamic resistance is alout $25 \Omega$, rising to about $25 \mathrm{k} \Omega$ for $I=1 \mu \mathrm{A}$. Log amplifiers have the best logaithmic :esponse when $I$ is in the $1-\mu A_{1}$ range. A junction capacitance of about $5 \mathrm{p} 5$ thus limits the bandwidth $10=1 \mathrm{MHz}$ 
Recent advances in hybrid circuit design have led to the availability of inexpensive quasi-logarithmic-response amplifiers such as the Analog Devices AD 640.3 These amplifiers have bandwidths exceeding $100 \mathrm{MHz}$, and compress the detected rfenvelope signal in a series of nonlinear amplification stages, providing a quasilogarithmic response. Thus the AD 640 overcomes several of the limitations inherent in the bipolar junction log-amps, including the temperature dependence, the unipolar signal requirement, and the low attainable bandwidth. A log-ratio circuit using four Analog Devices AD 640 amplifiers is shown in Fig. 6.

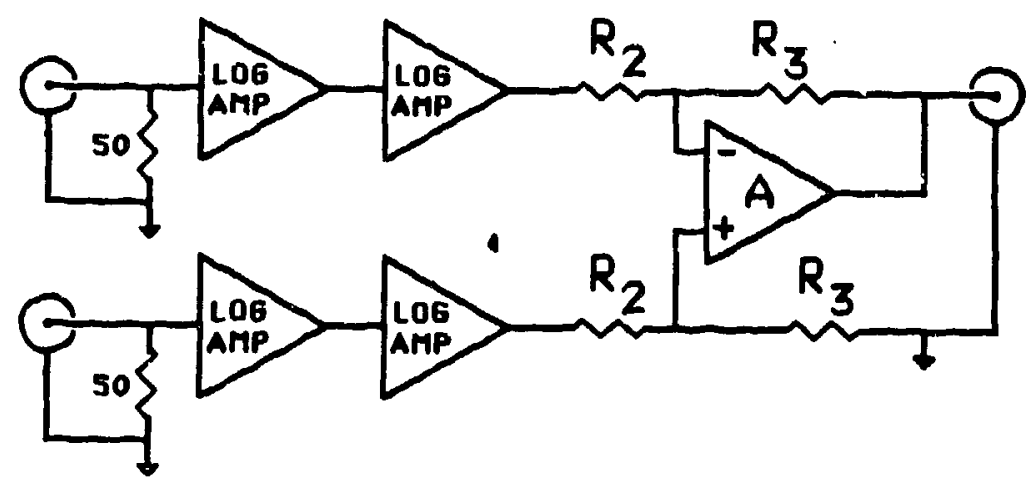

Fig.6. Block diagram of a log-ratio circuit using four Analog Devices AD 640 logamplifier hybrids.

The response uniformity of a circuit comprised of four Analog Devices AD 640s at $60 \mathrm{MHz}^{4}$ is shown in Fig. 7. The 21 curves represent input disparities from -10 to $+\mathrm{i} 0 \mathrm{~dB}$ in $1-\mathrm{dB}$ steps. Two amplifier chips are required on each input in order to achieve the large dynamic range. The observed response uniformity over a 60 -dB dynamic range is about $\pm 0.1 \mathrm{~dB}$. The ripple structure observed for off-center beams in Fig. 7 is due to the successive saturation of the various gain stages in the AD 640. The effect is minimized for centered beams, because the log-amplifiers on the two inputs track one another.

The expected resolution for beam position signal processing circıits is given by the relation, using Eq. (1-3).

$$
\frac{\delta x}{b}=\frac{1}{4}\left[\frac{\mathrm{KTBE}}{P_{\mathrm{in}}}\right]^{1 / 2}
$$

where $\delta x$ is the resolution, $b$ is the half aperture, $F$ is the electronics noise factor (not figure), kTB is the thermal noise power in bandwidth $B$, and $P_{\text {in }}$ is the signal power per electrode. The measured noise figure for the AD $640 \mathrm{log}$-ratio circuit in a $220 \mathrm{kHz}$ bandwidth at $60 \mathrm{MH}$ ? is about $15 \mathrm{~dB}^{4}$ (corresponding to a noise factor of 32), as shown in Fig. 8.

Higher frequency log-ratio operation is possible with any of several Plessey hybrid circuits, ${ }^{5}$ at considerably higher cost. Figure 9 shows the response uniformity of four Plessey SL3522A chips at $425 \mathrm{MHz} .^{6}$ A'though the individual Plessey chips have the 
required dynamic range, the ripple effect is very large, requiring the amplifiers on each input to be paralleled with a small gain offset between them.

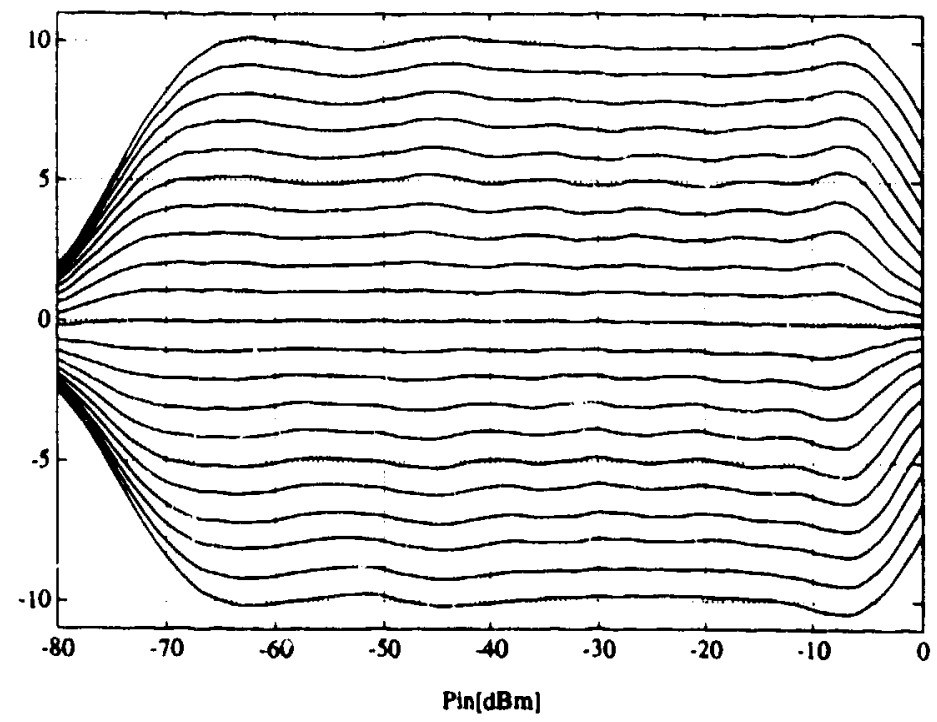

Fig. 7. Response uniformity for the circuit in Fig. 6 operating at $60 \mathrm{MHz}$. The horizontal axis is the signal input power in $\mathrm{dBm}$. The vertical axis is the signal output voltage. The cirves represent the amplitude disparity between inputs, ranging from -10 to $+10 \mathrm{~dB}$ in $1-\mathrm{dB}$ steps. Data is from $\mathrm{R}$. Aiello (see paper in these Proceedings).

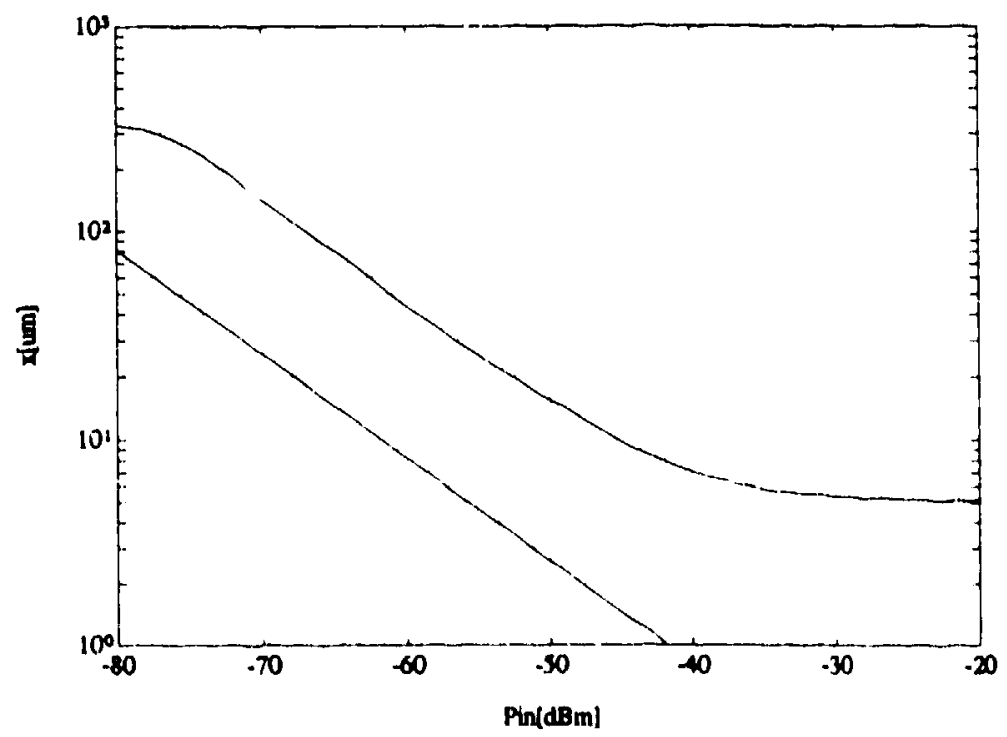

Fig. 8. Plot of the expected resolution for $a b=33-\mathrm{mm}$ half-aperture BPM vs. signal power for the AD $640 \mathrm{log}$-ratio amplifier circuit at $60 \mathrm{MHz}$ with a $220-\mathrm{kHz}$ bandwidth, and for an ideal circuit with only kTB noise. The amplifier noise figure is estimated to be about $15 \mathrm{~dB}$. Data trom R. Aiello (these Proceedings). 


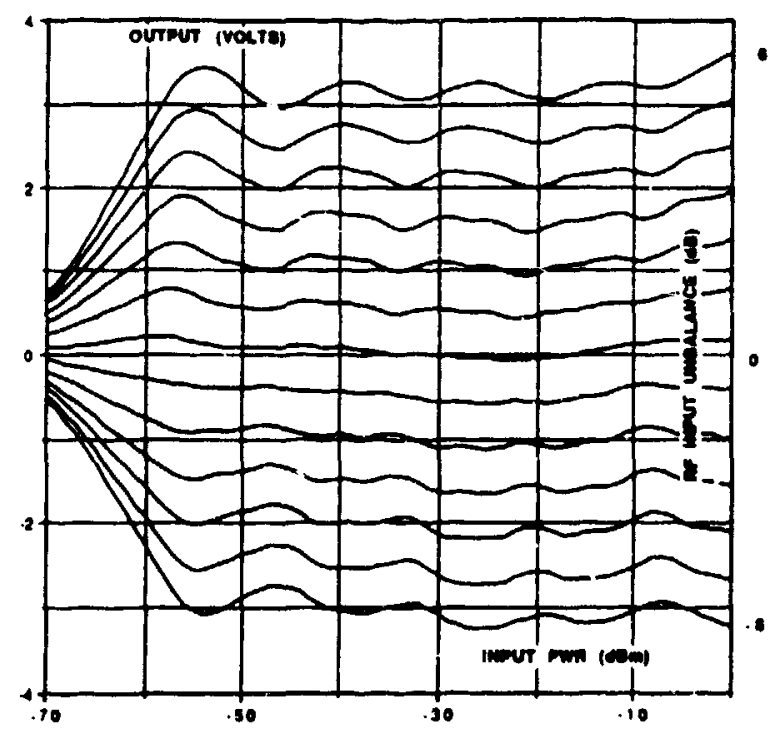

Fig. 9. Response uniformity of a log-ratio circuit using four Plessey SL3522A chips operating at $425 \mathrm{MHz}$. Data from F. D. Wells (LANL).

\section{SUMMARY}

The following Table summarizes some of the basic features of the three types of BPM signal processing.

Eearure

Time-domain processing

Frequency-domain processing

Carrier frequency range

Carier frequency slewing

Acquisition band width

Amplitude dynamic range

Displacement response linearity

Normalized real-time response

Cable phase-matching required

Amplifier noise figure

Cost

Circuit complexity

$\begin{array}{lll}\text { Difference-over-sum } & \text { AM-PM } & \text { Logratio } \\ \text { yes } & & \\ \text { yes } & \text { no } & \text { no } \\ >1 \mathrm{GHz} & \text { yes } & \text { yes } \\ \text { yes } & <100 \mathrm{MHz} & <500 \mathrm{MHz} \\ >100 \mathrm{MHz} & \text { no } & \text { yes } \\ \text { low } & \text { high } & \text { high } \\ \text { intermediate } & \text { poorest } & \text { best } \\ \text { no } & \text { yes } & \text { yes } \\ \text { depends } & \text { yes } & \text { no } \\ \text { can be very low } & >20 \mathrm{~dB} \text { typ } & 15 \mathrm{~dB} \text { achieved } \\ \text { lowest } & \text { highest } & \text { intermectiace } \\ \text { lowest } & \text { highest } & \text { intermediate }\end{array}$

In summary, the recent availability of hybrid logarithmic amplifiers have provided system designers a new option in BPM system design.

\section{REFERENCES}

1. R. Shafer, "Beam Position Monitoring," in AIl' Conf.Proc. "212, page 26 (1989).

2. R. Shafer, S. Jachim, and R. Webber, "AM-PM Position Measurement Circuit for the Tevatron," IEEE Trans. Nucl. Sci., Vol NS-28, Page 2323 (1981).

3. Analog Devices Corp. Cambridge, MA 02139.

4. R. Aiello, these Proceedings.

5. Plessey Corp,

6. F. D. Wells, private communication (1992). 